\title{
Altered pupillary size and darkness and light reflexes in Alzheimer's disease
}

\author{
R Prettyman, P Bitsios, E Szabadi
}

\begin{abstract}
The purpose was to compare resting pupil diameter in darkness and light, and the pupillary darkness and light reflexes between a group of patients with Alzheimer's disease and a group of healthy old people. Nine medication free patients with Alzheimer's disease and nine healthy control subjects, matched for sex and age with the patients, participated. There were six men and three women and the median age was 72 years in both groups. Pupil diameter was monitored with an infrared television pupillometer. Resting pupil diameter was smaller in the Alzheimer's disease group $(P=0 \cdot 041$, in darkness). The amplitude and the maximum dilatation velocity of the darkness reflex were smaller for the Alzheimer's disease group (maximum dilatation velocity $P<0.002)$. The amplitude and the $75 \%$ recovery time of the light reflex response were reduced in the Alzheimer's disease group $(P<0.002$ and $P=0.034$ respectively). There was no difference in the latency of the reflex response between the two groups. The reduced pupil size and diminished darkness reflex in the Alzheimer's disease group are consistent with a sympathetic deficit in the patients. The reduction in light reflex amplitude and recovery time are likely to be secondary to the grossly diminished pupil size in the patient group. The lack of any change in light reflex latency in the patients with Alzheimer's disease argues against an afferent defect. The pupillary changes in patients with Alzheimer's disease are qualitatively the same as those seen in healthy old people and are consistent with the notion of "accelerated aging" in Alzheimer's disease.
\end{abstract}

\section{(F Neurol Neurosurg Psychiatry 1997;62:665-668)}

Keywords: Alzheimer's disease; pupillary darkness reflex; pupillary light reflex; aging

The characteristic physiological and neuropathological changes seen in Alzheimer's disease $^{1}$ are often qualitatively similar to, but quantitatively more profound, than the alterations associated with aging. ${ }^{23}$ These findings have led to the notion that, in many respects, Alzheimer's disease represents the acceleration and accentuation of the normal aging process. ${ }^{24}$ Among the recognised clinical characteristics of normal aging are alterations in the function of the autonomic nervous system. ${ }^{56}$ Of particular interest are pupillary changes: pupil size declines linearly with age starting as early as the age of $20 .^{7}$

In a previous study ${ }^{8}$ we compared resting pupil size and the kinetic variables of the darkness and light reflexes between an elderly group and a group of young healthy people. We found smaller pupil sizes, reductions in the amplitude and velocity of the darkness reflex, and a prolongation of the recovery time of the light reflex in the elderly group, consistent with a reduction in sympathetic activity in old age. In the present study we extended these findings by comparing the pupillary measures between a group of patients with Alzheimer's disease and an age and sex matched group of healthy subjects.

\section{Methods}

Nine unmedicated patients with probable Alzheimer's disease according to the NINCDS-ADRDA criteria ${ }^{9}$ and nine healthy elderly subjects, matched with the patients for sex and age, were included in the study. There were six men and three women in each group. Patients were consecutive referrals who satisfied the inclusion or exclusion criteria, and control subjects were recruited individually to obtain a sample that was matched for age and sex with the patient sample. The median and mean (SD) ages of the patients with Alzheimer's disease were 72 and $70 \cdot 2(5 \cdot 9)$ and those of the healthy elderly subjects were 72 and $70.5(5.9)$. The healthy elderly subjects were taken from a larger cohort of a study examining the effect of aging on pupil size and reflexes. ${ }^{8}$ Healthy subjects with no history or presence of disorders of the central or autonomic nervous systems, psychiatric illness, ocular pathology, or hearing problems were included; none of the subjects was taking any medication. Patients with Alzheimer's disease also satisfied these criteria, with the exception 


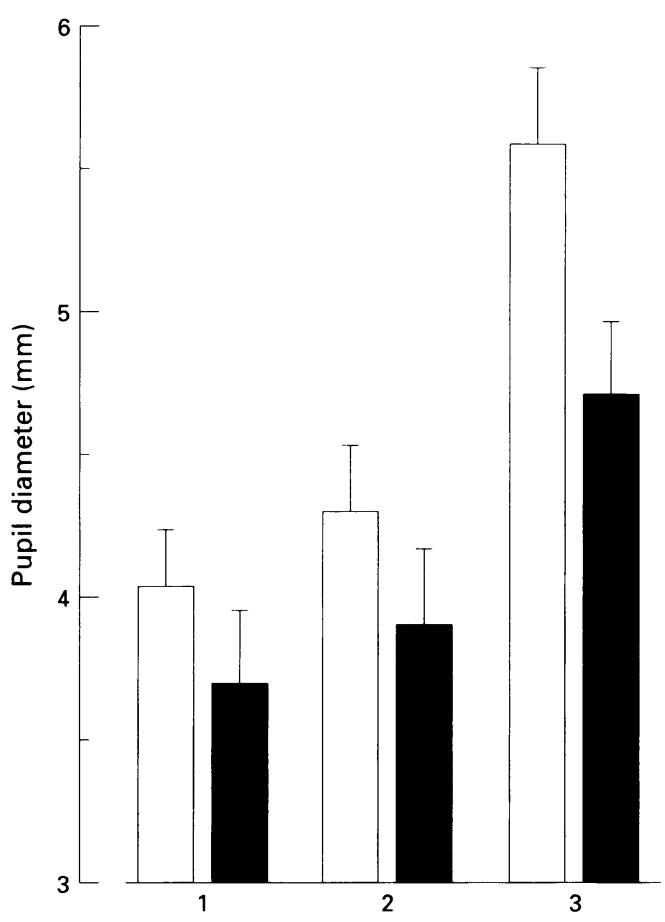

Figure 1 Resting pupil diameter under three illumination conditions. 1: $32 \mathrm{Cd} \mathrm{m}^{-2} ; 2: 16 \mathrm{Cd} \mathrm{m}^{2} ; 3$ : darkness (90 s after lights off). Open columns = healthy old subjects; closed columns = patients with Alzheimer's disease; vertical bars $=S E M$.

of the presence of the cognitive features of dementia associated with Alzheimer's disease. In addition to the above inclusion criteria, patients with Alzheimer's disease were selected on the basis of their ability to tolerate or cooperate with the experimental procedures. Patients with behavioural disturbance or severe dyspraxia or dysphasia were excluded from the study.

The general level of impairment of all the patients with Alzheimer's disease corresponded to the DSM-III-R criteria for dementia of either mild or moderate severity. Severity of the cognitive impairment was assessed with the mini mental state examination (MMSE) ${ }^{10}$ and the severity grades of Alzheimer's disease laid down by DSM-III-R. ${ }^{11}$ The mean (SD) MMSE score for the patients with Alzheimer's disease was $17.5(8.5)$ and it was 30.0 (the top score) for the healthy old subjects. The study was approved by the ethics committee of the Queen's Medical Centre NHS Trust, Nottingham.

Pupil diameter, under constant illumination and in response to sudden changes in the level of illumination to evoke the pupillary darkness and light reflexes, was monitored with an infrared binocular television pupillometer (Applied Science Laboratories, Waltham MA, USA), as described previously. ${ }^{8}$

Means (SD) were calculated for each pupillary variable within each of the two groups (patients and healthy old subjects). The distribution of each variable was assessed by calculating the skew and kurtosis indices. As in no case did either index deviate significantly from zero, parametric statistics were used. Resting pupil size in light and darkness and darkness reflex data were compared between the groups by unpaired $t$ tests. Light reflex data were analysed by two factor (group $\times$ light intensity) analyses of variance (ANOVA). The possible dependence of the kinetic measures on resting pupil diameter (in the dark) was investigated by Pearson's correlation coefficients.

\section{Results}

RESTING PUPIL DIAMETER

The resting pupil diameter was smaller in the patient group $(t=-2 \cdot 22, \mathrm{P}=0.041$, in the dark; fig 1). The mean differences $(95 \%$ confidence interval $(95 \% \mathrm{CI})$ ) between the controls and the patients were $(\mathrm{mm}): 32 \mathrm{Cd} \mathrm{m}^{-2}: 0.31$ $(-0.37$ to 1.00$) ; 16 \mathrm{Cd} \mathrm{m}^{2}: 0.39(0.36$ to 1.13); darkness: $0.83(0.04$ to 1.63$)$.

\section{DARKNESS REFLEX}

There was a trend for smaller darkness reflex amplitudes $(\mathrm{t}=1.79 ; \mathrm{P}=0.09)$ and there was a significantly lower maximum dilatation velocity $(t=6.26 ; P<0.001)$ in the Alzheimer's disease group (fig 2A). The mean differences $(95 \% \mathrm{CI})$ between the controls and the patients were: amplitude (mm): $0 \cdot 07$ $(-0.21$ to $0 \cdot 16)$; maximum dilatation velocity $\left(\mathrm{mm} \mathrm{s}^{-1}\right): 0 \cdot 31(0 \cdot 21$ to $0 \cdot 41)$.

\section{LIGHT REFLEX}

The amplitudes and recovery times, at all light intensities, were reduced in the Alzheimer's disease group; latency was not significantly different between the two groups (fig 2B). There were significant correlations between resting pupil diameter in the dark and amplitude at all four light intensities $\left(\mathrm{mW} \mathrm{cm}^{-2}\right)$ : $8.5 \times 10^{-3}: r=0.529, \mathrm{P}=0.024 ; 7.0 \times$ $10^{-2}: r=0.663, \mathrm{P}<0.005 ; 0.43: r=0.759$, $\mathrm{P}<0.001 ; 1.84: r=0.835, \mathrm{P}<0.001$. Latency and $75 \%$ recovery time did not correlate significantly with resting pupil diameter in the dark at any of the four light intensities (latency: $r=-0.125, P=0.62 ; r=0.056$, $\mathrm{P}=0.83 ; \quad r=-0.139, \quad \mathrm{P}=0.58 ; \quad r=$ $-0 \cdot 206, \mathrm{P}=0.41 ; 75 \%$ recovery time: $r=$ $0.349, \mathrm{P}=0.16 ; r=0.305, \mathrm{P}=0.22 ; r=$ $0.208, \quad \mathrm{P}=0.41 ; \quad r=0.016, \quad \mathrm{P}=0.95)$. Analysis of variance showed significant main effects of light intensity $(F=114.75$; df $=$ $3,48 ; \mathrm{P}<0.001)$ and group $(F=13.23$; df $=$ $1,16 ; P<0.002)$, and no significant interaction $(F=1.15 ; \mathrm{df}=3,48 ; \mathrm{P}=0.34)$ for amplitude, and significant main effects of light intensity $(F=17.86 ; \mathrm{df}=3,48 ; \mathrm{P}<0.001)$ and group $(F=5.41 ; \mathrm{df}=1,16 ; \mathrm{P}=0.034)$ and no significant interaction for recovery time of the light reflex $(F=0.8 ; \mathrm{df}=3,48 ; \mathrm{P}=$ $0 \cdot 5)$. For latency, there was a significant main effect of light intensity $(F=13 \cdot 49$; df $=3,48$; $\mathrm{P}<0.001)$ but no significant main effect of group $(F=1.07 ; \mathrm{df}=1,16 \mathrm{P}=0.32)$ or interaction $(F=0.77 ; \mathrm{df}=3,48 \mathrm{P}=0.51)$.

\section{Discussion}

The size of the pupil at any one time reflects the relation between the opposing sympathetic (dilator) and parasympathetic (constrictor) influences. ${ }^{7}$ The kinetic variables of the dark- 
Figure 2 Pupillary darkness and light reflexes. (A) Darkness reflex. Top: example of a response showing the kinetic variables measured. Ordinate = pupil diameter $(\mathrm{mm})$, abscissa $=$ running time $(s)$, horizontal bar $=$ darkness stimulus. The variables measured were amplitude (mm) and maximum dilatation velocity $(\mathrm{mm}$ $\left.s^{-1}\right)$ measured at the steepest segment of the dilatation curve. Bottom: Comparison of the parameters of the darkness reflex between healthy old subjects (open columns) and patients with Alzheimer's disease (closed columns). Left: amplitude (mm), right: maximal dilatation velocity $(\mathrm{mm}$ $s^{-1}$. Vertical bars over the columns are SEM. (B) Light reflex. Top:

$A$ response showing the kinetic parameters measured. Ordinate $=$ pupil diameter $(\mathrm{mm})$, absciss $a=$ running time (s), horizontal bar = light stimulus. The variables measured were latency $(s)$, amplitude ( $\mathrm{mm})$, and $75 \%$ recovery time (s).

Bottom: Comparison of the variables of the light reflex, evoked by four stimuli of increasing intensity, between healthy old subjects (open symbols) and patients with Alzheimer's disease (closed symbols). Vertical bars are SEM.
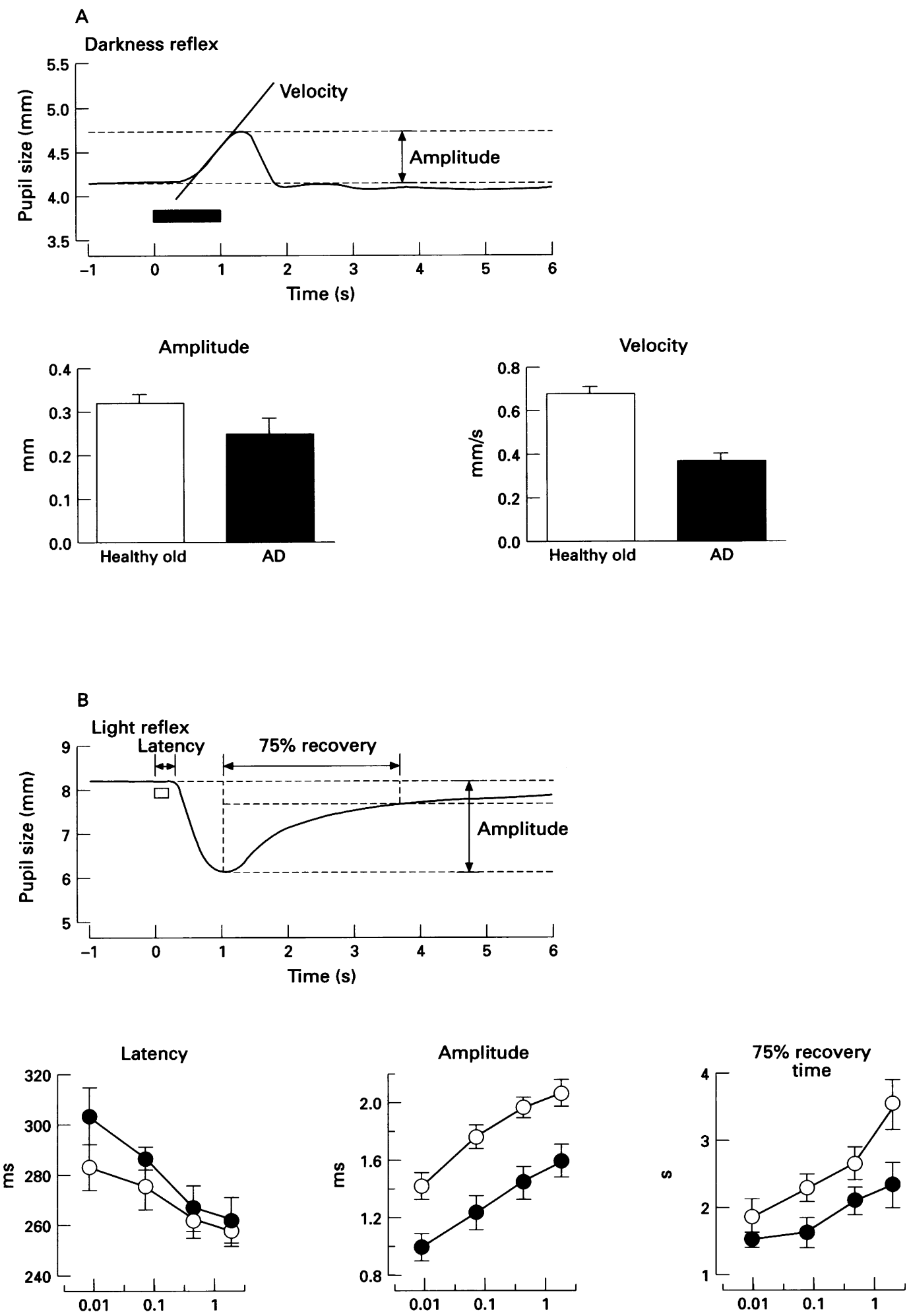

Light intensity $\left(\mathrm{mW} \mathrm{cm}^{-2}\right)$ ness and light reflexes enable us to dissect the relative contributions of these influences. The darkness reflex and the recovery time of the light reflex reflect mainly sympathetic activation, whereas the latency and amplitude of the light reflex are governed by parasympathetic activity. ${ }^{712}$

In an earlier study ${ }^{8}$ we have found that healthy old people, in comparison with younger subjects, show reduced pupil diameters together with reductions in the variables of the darkness reflex, consistent with a possible sympathetic deficit. In the present study, the patients with Alzheimer's disease, compared with healthy old people, showed a similar deficit, in agreement with the model of "accelerated aging" in Alzheimer's disease. We suggest that this sympathetic deficit, both in healthy old people and patients with Alzheimer's disease, may be related to the loss of neurons in the locus coeruleus and other noradrenergic nuclei in the brain stem from which sympathoexcitatory influences descend to the spinal cord. ${ }^{13}$ Indeed, there is evidence that the monotonic decline in pupil size with $\mathrm{age}^{7}$ is paralleled by a decline in cell number in 
the locus coeruleus. ${ }^{14}$ Furthermore, there is a severe loss of neurons in the locus coeruleus in Alzheimer's disease which is more pronounced than that seen in healthy old people. ${ }^{3}$

As the patients with Alzheimer's disease had very small pupil sizes, it is difficult to interpret the light reflex data in terms of autonomic activity. Both the reduced amplitude and recovery time may be explained by the curtailment of the responses by a "floor effect". 1215 Therefore, it is difficult to draw any conclusions about the possible involvement of the parasympathetic innervation of the iris in Alzheimer's disease. Whereas the reduction in resting pupil size would be consistent with an enhancement of the parasympathetic input, the reduction in amplitude points towards a possible reduction in parasympathetic tone. It has been reported that patients with Alzheimer's disease show increased sensitivity to the mydriatic effect of tropicamide, ${ }^{16}$ a muscarinic receptor antagonist, consistent with the notion of increased parasympathetic tone to the iris. A possible mechanism for this may be parasympathetic disinhibition due to the loss of the noradrenergic inhibitory control of the Edinger-Westphal nucleus from the locus coeruleus. ${ }^{13}$ However, the effect of tropicamide in patients with Alzheimer's disease is controversial: whereas some investigators found a trend towards increased sensitivity in Alzheimer's disease, ${ }^{17} 18$ others failed to confirm it. ${ }^{19} 20$ On the other hand, the recent finding, in one study, of increased sensitivity of patients with Alzheimer's disease to the miotic effect of the cholinoceptor agonist pilocarpine $^{21}$ is consistent with the notion of denervation supersensitivity, indicating a possible parasympathetic deficit.

As patients with Alzheimer's disease may have degenerative changes in the retina ${ }^{22}$ and optic nerve, ${ }^{23}$ the possibility should be considered that some of the pupillary changes may have been due to an "afferent pupillary defect". However, this is unlikely as patients with Alzheimer's disease do not show this abnormality on neuro-ophthalmological examination. ${ }^{24}$ Furthermore, the afferent pupillary defect is characterised by elongation of light reflex latency and normal or slightly dilated pupils; $;^{25}$ however, our patients showed unchanged reflex latencies and constricted pupils. It should be noted, however, that as the present results were obtained in a small sample of subjects, the power to reject the null hypothesis was relatively low, and therefore non-significant differences, as in the case of light reflex response latency, may still be of clinical relevance. Further studies with a larger cohort of patients and control subjects are needed to confirm the present results.

PB was supported by a scholarship from the State Scholarship Foundation of Greece (IKY).

1 Khachaturian ZS. Diagnosis of Alzheimer's disease. Arch Neurol 1985;42:1097-105.

2 Mann DMA. The neuropathology of Alzheimer's disease: a review with pathogenetic, aetiological and therapeutic considerations. Mech Ageing Dev 1985;31:213-55.

3 Palmer AM, DeKosky ST. Monoamine neurons in aging and Alzheimer's disease. $\mathcal{F}$ Neural Transm 1993;91: 135-59.

4 Newton RD. The identity of Alzheimer's disease and senile dementia and their relationship to senility. $\mathcal{F}$ Ment $S c i$ 1948;94:225-9.

5 O'Brien IAD, O Hare P, Corrall RJM. Heart rate variability in healthy subjects: effect of age and the derivation of normal ranges for tests of autonomic function. $\mathrm{Br} \mathrm{Heart} f$ 1986;55:348-54.

6 Lamb K, Bradshaw CM, Szabadi E. The responsiveness of human eccrine sweat glands to choline and carbachol: application to the study of peripheral cholinergic funcapplication to the study of peripheral cholinergic func1983;24:55-62.

7 Smith SA. Pupil function: tests and disorders. In Bannister R, Mathias CJ, eds. Autonomic failure. 3rd ed. Oxford: Oxford University Press 1992:393-412.

8 Bitsios P, Prettyman R, Szabadi E. Changes in autonomic function with age: a study of pupillary kinetics in healthy young and old people. Age Ageing 1996;25:432-8

9 McKhann G, Drachman D, Folstein M, et al. Clinical diagnosis of Alzheimer's disease: report of the NINCDS ADRDA Work Group under the auspices of Department of Health and Human Sevices Task force on Alzheimer's Disease. Neurology 1984;34:939-44.

10 Folstein MF, Fostein SE, McHugh PR. Mini mental state: a practical method for grading the cognitive state of practical method for grading the cognitive state of

11 American Psychiatric Association. Diagnostic and statistical manual of mental disorders. 3rd ed, revised (DSMcal manual of mental disorders. 3rd

12 Theofilopoulos N, McDade G, Szabadi E, et al. Effects of reboxetine and desipramine on the kinetics of the pupillary light reflex. Br f Clin Pharmacol 1995;39:251-5.

13 Szabadi E, Bradshaw CM. Autonomic pharmacology of $\alpha_{2}$ adrenoceptors. $\mathcal{f}$ Psychopharmacol 1996;10(suppl 3): 6-18

14 Manaye KF, McIntire DD, Mann DMA, German DC. Locus coeruleus cell loss in the aging human brain: non-random process. $f$ Comp Neurol 1995;358:79-87.

15 Longmore J, Theofilopoulos N, Szabadi E, et al. Modification of the pupillary light reflex by miotic and mydriatic drugs: applicability of model of functional interaction. Brf Clin Pharmacol 1987;23:610-1P.

16 Scinto LFM, Daffner KR, Dressler D, et al. A potential non-invasive neurobiological test for Alzheimer's disease. Science 1994;266:1051-4.

17 Gomez Tortosa E, Del Barrio A, Jimenez Alfaro I. Pupil response to tropicamide in Alzheimer's disease and other neurodegenerative disorders. Acta Neurol Scand 1996; 94:104-9.

18 Arai $\mathrm{H}$, Terajima $M$, Nakagaw $T$, et al. Pupil dilatation assay by tropicamide is modulated by apolipoprotein $\mathrm{E}$ $\varepsilon 4$ allele dosage in Alzheimer's disease. Neuroreport 1996 7:918-20.

19 Loupe DN, Newman NJ, Green RC, et al. Pupillary response to tropicamide in patients with Alzheimer disease. Ophthalmology 1996;103:495-503.

20 Treloar AJ, Assin M, MacDonald AJD. Pupillary response to topical tropicamide as a maker for Alzheimer's disease. Br 7 Clin Pharmacol 1996;41:256-7.

21 Idiaquez J, Alvarez $\mathrm{G}$, Villagra $\mathrm{R}$, et al. Cholinergic supersensitivity of the iris in Alzheimer's disease. $\mathcal{F}$ Neurol sensitivity of the iris in Alzheimer'
Neurosurg Psychiatry 1994;57:1544-5.

22 Bassi CJ, Blanks JC, Sadun AA, et al. The retinal ganglion cell layer in Alzheimer's disease: a whole mount study. cell layer in Alzheimer's disease: a whol
Invest Ophthalmol Vis Sci 1987;28:109.

23 Hinton DR, Sadun AA, Blanks JC, et al. Optic nerve degeneration in Alzheimer's disease. N Engl $\mathcal{F}$ Med 1986, 315:485-7.

24 Sadun AA, Borchert M, DeVita E, et al. Assessment of visual impairment in patients with Alzheimer's disease. Am f Ophthalmol 1987;104:113-20.

25 Cox TA. Pupillography of a relative afferent pupillary defect. Am f Ophthalmol 1986;101:320-4. 